\title{
Delirium in acute neurological care department. Causes, pathogenesis and management
}

\author{
Iulian Dan Cuciureanu², Cristina Croitoru², Victor Constantinescu' ${ }^{1}$, \\ Tudor Cuciureanu' 1 \\ 1"Gr. T. Popa" University of Medicine and Pharmacy, Iasi , Romania \\ 2"Prof. Dr. N. Oblu” Emergencies Clinical Hospital, lasi, Romania
}

\begin{abstract}
Defined as an organic decline, often transient, undulant, mainly of cognitive function, memory, language, delirium is often considered the attribute of patients hospitalized with multiple illnesses. Medical costs, and subsequent psycho-logical implications, prognostic particularities gives him a special social importance. Although with multiple recog-nized etiology, its pathogenesis is partially known, involving relays and neural networks. In the presence of known predisposing factors and under some precipitating influence like acute metabolic or inflammatory disorders (interleu-kins 1, 6, caspaze), infections, vascular endothelial dysfunction that cause malfunctions of neurotransmitters (GABA, glutamate, acetylcholine, melatonin) occurs, mainly, an attentional matrix disruption (attention modulation is done through the collaboration of the neocortex, thalamus and brainstem's reticular substance) as well as structural chang-es of neural networks and their interaction with cerebral default network. Its prevalence is estimated at the time of admission between $11-30 \%$ of patients. Early identifi cation of patients at risk and multifactorial and multidisciplinary approach represents efficient solution to prevent delirium consequences. Authors performed a synthetic rundown of the main theoretical aspects of delirium and exemplify by a retrospective study in "Prof. Dr. N. Oblu" Neurological Clinic of Emergencies hospital, lasi. From 3,707 patients admitted in one year, $32 \%$ had impaired language and only $7 \%$ have presented confusional syndrome: $58 \%$ in ischemic strokes or hemorrhagic $(9 \%)$, epilepsy $(18 \%)$, intra-cerebral tumors $(1.8 \%)$, metabolic encephalopathies $(7 \%)$, neurodegenerative disorders (22\%), infections (3\%). Ages prevailed over 60 years and males $(64 \%)$, low outcome in $15 \%, 80 \%$ had improvement in evolution and $5 \%$ died.
\end{abstract}

Keywords: delirium, causes, management

\section{INTRODUCTION}

Delirium is also known throughout medical literature as acute confusional state, organic psychosis, acute organic brain syndrome, acute cerebral insufficiency or toxic-metabolic encephalopathy (10), all reflecting the word's etymology. Delirium derives from the latin word "delirare" which can be literally translated into "deviate from the furrow".

In a much more complex definition of the term, delirium is an acute, fluctuating, transient, usually reversible neuropsychiatric syndrome characterized by two main features: cognitive dysfunctions and inattention, the last being a sine qua noncondition (10).
Delirium can occur at any age; however it is a frequent cause and an important complication of hospitalization especially among elderly people (10). In studies where patients were assessed within 24 hours, prevalence rates ranged from $10 \%$ to $31 \%$ among older hospitalized patients $(26,21)$.

Even though it is defined as a reversible mental disorder, delirium can become chronic with permanent functional and cognitive sequelae (16). Predominantly in the elderly, it can be the trigger factor for poor outcomes, irrespective of the underlying cause: functional decline, loss of independence, increased hospitalization (10) and rapid progression of underlying dementia (9). Death occurs in an 
approximately quarter to third of patients $(33,23)$ and it is due either to the underlying condition that precipitated delirium or to complications secondary to prolonged in bed immobilization.

Despite the fact that approximately $30-40 \%$ of delirium cases are preventable (26), there is an increased rate of under diagnosis. This is an secondary to incomplete understanding of pathogenic mechanisms, difficulty in recognizing the symptoms as delirium, lack of biological markers and limited pharmacological therapy.

Taking into consideration the fact that early identification of patients at risk and multifactorial and multidisciplinary approach represents efficient solution to prevent its consequences, the authors performed a rundown of the main synthetic theory of delirium. Therefore, the most frequent causes, pathogenic mechanisms and main principles of prevention and management are discussed and then illustrated by a retrospective study in "Prof. Dr. N. Oblu” I Neurology Clinic of Emergency Clinical Hospital.

\section{MATERIALS AND METHOD}

The authors performed a retrospective clinical study of 3707 patients admitted at the Iasi "Prof. Dr. N. Oblu" Emergency Clinical Hospital, neurological clinics, between 1012015 and 31122015 . The data were collected from the clinic's medical records, emergency medical records and intensive care charts. Clinical information registered included: diagnosis at admission, diagnosis at 72 hours and diagnosis at release from hospital, age and gender psychological assessment using Mini Mental State Examination (MMSE) and Global Assessment of Functioning Scale (GAFS). Statistical analysis of data obtained was then performed.

\section{RESULTS}

From the 3,707 patients admitted $7 \%$ presented acute confusional non traumatic state characterized mainly by psychomotor involvement and impaired cognition, with slowly or no response to questioning but also by psychomotor agitation, excitation and hyper vigilance. Age over 60 years prevailed as well as male sex (60\%). Regarding the main pathology, $39 \%$ of delirium cases occurred secondary to an ischemic stroke, while $9 \%$ to a hemorrhagic one. Neurodegenerative pathology was found in $22 \%$ of patients. Epilepsy was the cause of either ictal or postictal delirium in $18 \%$ of cases. Other causes were less frequent: metabolic encephalopathy $(7 \%)$, infections $(3 \%)$ or cranial space occupying lesions $(1,8 \%)$.

Regarding the short time outcome, $80 \%$ of patients improved, $15 \%$ had no change in general condition and 5\% deceased due to underlying disease.

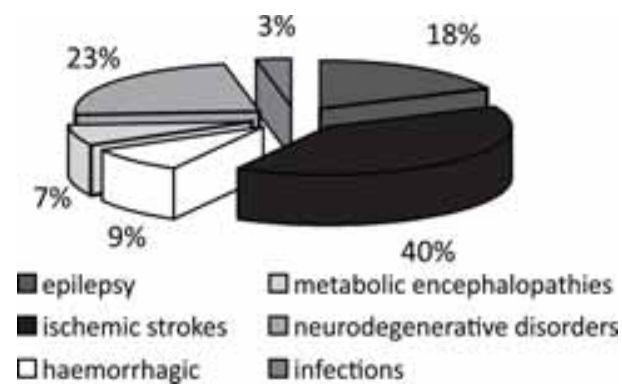

\section{DISCUSSIONS}

Delirium is clinically characterized by subacute changes in cognition, awareness, behavioral disturbances, inversion of the sleep-wake cycle and also illusions and simple hallucinations. The two common features of these manifestations are fluctuation during the course of the day and nocturnal exacerbation (19). In many reviews of medical literature, delirium is divided into three categories: hyperactive secondary to alcohol, benzodiazepine or barbiturate withdrawal (14), hypoactive witch is the most common form in elderly patients and mixed syndromes. It appears that hyperactive delirium has the best prognosis (18).

Delirium is a multi-factorial mental disorder that results from the attack of multiple possible trigger factors upon an individual that possess numerous predisposing/risk factors. Risk factors are classically divided into modifiable (sensory impairment, immobilization, polypharmacy, environment, sustained sleep deprivation, emotional distress or alcohol excess) and nonmodifiable (age over 65 years, male sex, history of delirium, stroke, neurological disease, falls or gait disorder, multiple comorbidities) (10).

The most studied non modifiable risk factor that has shown the highest cause-effect association is 
preexisting dementia (3). At least two thirds of cases of delirium occur in patients with underlying dementia or cognitive impairment (13). It appears that vascular dementia and late-onset Alzheimer disease predispose to delirium more than fronto temporal dementia (24).

The results of the present study correspond to the literature: male predominance and advanced age are known non modifiable risk factors. Neurodegenerative diseases were found in $22 \%$ of delirium cases. This is consistent with the fact that synaptic loss and neuron destruction increase the vulnerability of the nervous system to trigger factors, by altering the "brain reserve" (7). It is also interesting to mention that the relation between delirium and dementia is bilateral: an acute confusional state could contribute to developing dementia in the future. Also, delirium may unmask a previously "mute" dementia. However, by Diagnostic and Statistical Manual of Mental Disorders, Fifth Edition (DSM5) criteria, dementia cannot be diagnosed with certainty when delirium is present (1).

In terms of trigger factors, acute confusional states have six major causes: toxic-metabolic encephalopathies, multifocal brain lesions (including neurodegenerative disorders and infections of the central nervous system) which produce multiple small neuronal lesions, focal brain lesions especially secondary to ischemic stroke, head trauma lesions which produce a disturbance in the structure of cortex/midbrain/brainstem, space-occupying lesions and epilepsy (19).

Theoretically any metabolic disturbance that modifies acid/base balance, electrolyte balance or nutritional requirements of both neurons and glia can interfere with neurotransmitter action: acidosis, alkalosis, hyponatremia, hypernatremia (31), hyperglycemia, hypoglycemia, renal insufficiency, hepatic failure, anemia, endocrinopathies (thyroid storm, Hashimoto's encephalopathy), hypoxia/anoxia, infection and paraneoplastic states. In this category are also included both alcohol, barbiturates, opiates withdrawal and administration of various medication especially drugs that interfere with the cholinergic transmission: mainly antiparkinsonian medication (levodopa) but also neuroleptics, antidepressants and antihistamines (19).

One of the most frequent space-occupying lesion that produces delirium in elderly people is a chronic subdural hematoma which can appear following a minor trauma of a brain with increased venous fragility and generalized brain atrophy. Also, primary or secondary brain tumors may manifest with delirium. $78 \%$ of patients have psychiatric symptoms during brain tumor development, but only $18 \%$ of these present with isolated psychiatric symptoms as the first clinical manifestation (15). A recent case report states delirium as the first presenting syndrome of melanoma brain metastases (20). The low prevalence of space-occupying lesions as a cause of delirium in the present study may be explained by the fact that most space-occupying lesions are admitted in a neurosurgery service and not in a neurology clinic.

The connection between delirium and epilepsy is quite complex due to the fact that these conditions are often intertwined: absence or complex partial seizures can mimic delirium, many of the conditions resulting in delirium may also induce seizures and both conditions may coexist at the same patient (14). Epileptic seizures are due to and also produce a disturbance in the electrical network of the cortex and lower central nervous structures which may induce delirium. In relation to a seizure, delirium can be ictal or postictal. Ictal delirium can occur during repeated or prolonged absence seizures or complex partial seizures. Post ictal delirium refers to cortical impairment lasting for 4 to 10 days after a cluster of seizures (2). It is important to distinguish postictal delirium from postictal psychosis which is characterized by religious delusions and feelings of mystic fusion with the universe or from chronic interictal psychosis which is characterized by mainly auditive hallucinations (6).

In terms of delirium and stroke, the two pathological entities are frequently associated, sometimes delirium being the only presenting feature of a stroke in common investigation schedule. The incidence of delirium in the acute phase of stroke varied from $13 \%$ to $48 \%$ and was higher in elderly patients (22). Given the fact that there have been only a limited number of studies that have assessed delirium post-stroke (18), it is not clear whether delirium is a direct consequence of stroke or if it is due to stroke complications (infections, aspiration). However, delirium was more common after stroke than after acute coronary symptoms (13\% versus $2 \%$ ), indicating that it might be more related to 
brain damage than complications of prolonged in bed immobilization (22).

It appears that onset of delirium is dependent on specific features of the stroke: type of stroke - more frequent in intracerebral hemorrhages (4) and in cardioembolic stroke or total anterior circulation infarction (27); extent of stroke, the extent of cerebral edema post-stroke and extent of cerebral hypoperfusion. In terms of specific area of brain affected by stroke, several studies have shown that delirium is more frequent in left-sided strokes (11), unilateral lesions in the parahippocampal-fusiform-lingual gyri on either side of the brain, infarcts of posterior parietal and inferior prefrontal regions in the right hemisphere (19), lesions in the thalamus and caudate nucleus (18).

In the present study, $39 \%$ of delirium cases occurred secondary to an ischemic stroke, while $9 \%$ to a hemorrhagic one. These results are consistent with the ones in the medical literature, confirming once again that stroke is a well known precipitating factor for delirium (8).

The similar clinical presentation of delirium suggests common pathogenesis regardless of multiple predisposing and precipitating factors. Although pathogenic mechanisms are not entirely known (34) there are some generally accepted theories. The matrix of attention is severely impaired by disruption of either neurotransmission or connections of the neuronal network. Several mechanisms are involved: elevated levels of cortisol associated with acute stress response (29), endothelial dysfunction secondary to impaired auto regulation of cerebral blood flow (25) or increase in pro inflammatory cytokines such as interleukin- $1 \beta$ generated through adenosine-dependent activation of caspase 1 after reoxygenation (5).

The main hypothesis is reversible impairment of cerebral oxidative metabolism and multiple neurotransmitter abnormalities (35). From all neurotransmitters, lack of acetylcholine is the most studied in delirium (33). Pro arguments are the facts that anticholinergic drugs can cause delirium in susceptible patients and that central cholinesterase inhibitors, such as Donepezil can improve delirium in this case (10). Also, an imbalance between acetylcholine and dopamine, with the excess of the latter can cause delirium. That is why antiparkinsonian drugs may cause symptoms of delirium and also haloperidol, a dopamine antagonist is effective in controlling them (18). Decreased gamma-aminobutyric acid activity has been implicated in delirium from sedative drug-withdrawal or toxic delirium (30). Other neurotransmitters also appear to be implicated: glutamate, 5-hydroxytryptamine, beta-endorphin (10).

Direct injury of neuronal a network can be caused by multiple metabolic insults. Lesions that interfere with the bottom-up or top-down regulation of attention or disruption of intentional modulations gives rise to the clinical syndrome of acute confusional states (19). Also, lesions in the default memory network can cause delirium, especially in the dorsal anterior cingulate cortex which is a node that modulates attention, motivation, error detection and network memory (32).

Regarding management and prevention, to date, there is no unanimous protocol. However, non pharmacological measures targeting modifiable predisposing factors can significantly reduce the incidence of delirium (12). Pharmacological prevention may include haloperidol administration which can reduce the severity and duration of symptoms, but not for routine prophylaxis (10).

Non pharmacological strategies are the first line of treatment for all patients with delirium: nursing in a good sensory environment, avoiding physical restraints, preventing pressure sores and malnutrition (18). Current pharmacological strategies are largely based on case series and retrospective studies and seem to differ accordingly to the clinical form of delirium (10). For acute agitation, haloperidol is the drug of choice, although the evidence-base for this is weak (17). Moreover, antipsychotics increase the risk of stroke in elderly patients with dementia and also can prolong QT interval (10). Cholinesterase inhibitors may be used in the future. Benzodiazepines are not recommended as first-line agents because they may induce over sedation. They are reserved for treatment of drug withdrawal, diffuse Lewy body disease or second-line treatment of delirium (10).

Overall, the presence of delirium increases the medical costs. It determines increased number of hospitalization days and higher per day hospital losses resulting in a substantial economic burden.

Many avenues of future research exist in the complex field of delirium: unmasking complete 
pathogenesis, developing new screening tools and biological markers. All in order to prevent and manage delirium, as a method of increasing quality of life of patients and lowering the medical economic burden.

Conflict of interest: none declared Financial support: none declared

\section{REFERENCES}

1. American Psychiatric Association. Diagnostic and Statistical Manual of Mental Disorders, Fifth Edition. 5th ed. Washington, DC: American Psychiatric Association; 2013.

2. Biton V., Gates J.R., DePadua S.L. Prolonged postictal encephalopathy. Neurology. 1990; 40: 963-966.

3. Bruce A.J., Ritchie C.W., Blizard R., Lai R., Raven P. The incidence of delirium associated with orthopedic surgery: a meta-analytic review. Int. Psychogeriatr. 2007; 19: 197-214.

4. Caeiro L., Ferro J., Albuquerque R., Figueira M.L. Delirium in the first days of acute stroke. J Neurol. 2004; 251: 171-178.

5. Chiu G.S., Chatterjee D., Darmody P.T., Walsh J.P., Meling D.D., Johnson R.W., Freund G.G. Hypoxia/reoxygenation impairs memory formation via adenosine-dependent activation of caspaze 1. J Neurosci. 2012; 32(40): 13945-13955.

6. Croitoru C.G., Rosu I., Turliuc S. From epilepsy to schizoform organic delusional disorder - a case report of chronic interictal psychosis. Bulletin of Integrative Psychiatry. 2017; 2(73): 97-103.

7. Davis D.H.J., Skelly D.T., Murray C., Hennessy E., Bowen J., Norton S., Brayne C., Rahkonen T., Sulkava R., Sanderson D.J., Rawlins N., Bannerman D.M., MacLullich A.M.J., Cunningham C. Worsening cognitive impairment and neurodegenrative pathology Progressively increase risk for delirium. Am J Geriatr Psychiatry. 2015; 23(4): 403-415.

8. Ferro J.M., Caeiro L., Verdelho A. Delirium in acute stroke. Curr Opin Neurol. 2002; 15: 51-55.

9. Fong T.G., Jones R.N., Shi P., Marcantonio E.R., Yap L., Rudolph J.L., Yang F.M., Kiely D.K., Inouye S.K. Delirium accelerates cognitive decline in Alzheimer's disease. Neurology. 2009; 72(18): 1570-1575.

10. Fong T.G., Tulebaev S.R., Inouye S.K. Delirium in elderly adults: diagnosis, prevention and treatment. Nat Rev Neurol. 2009; 5(4): 210-220.

11. Gustafson Y., Olson T., Erikkson S., Asplund K., Bucht G. Acute confusional states (delirium) in stroke patients. Cerebrovasc Dis 1991; 1: 257-264.

12. Inouye S.K., Bogardus S.T., Charpentier P.A., Leo-Summers L., Acampora D., Holford T.R., Cooney L.M. Jr. A multicomponent intervention to prevent delirium in hospitalized older patients. N Engl J Med. 1999; 340: 669-676.

13. Inouye S.K., Ferrucci L. Elucidating the pathiphysiology of Delirium and the Interrelationship of delirium and dementia. J Gerontol A Biol Sci Med Sci. 2006; 61(12): 1277-1280.

14. Kaplan P.V. Delirium and epilepsy. Dialogues in Clinical Neuroscience. 2003; 5(2): 187-200.

15. Keschner M., Bender M.B., Strauss I. Mental symptoms associated with brain tumor: a study of 530 verified cases. JAMA. 1938; 110(10): 714-718.

16. Levkoff S.E., Evans D.A., Liptzin B., Cleary P.D., Lipsitz L.A., Welte T.T., Reilly C.H., Pilgrim D.M., Schor J., Rowe J. Delirium.
The occurrence and persistence of symptoms among elderly hospitalized patients. Arch. Intern. Med. 1992; 152:334-340.

17. Lonergan E., Britton A., Lixemberg J., Wyller T. Antipsyhotics for delirium. Cochrane Database Syst Rev. 2007; 18(2): CD005594

18. McManus J., Pathansali R., Stewart R., Macdonald A., Jackson S. Delirium post-stroke. Age and Aging. 2007; 36: 613-618.

19. Mesulam M.M. (2000). Principles of behavioral neurology. New York, US: Oxford University Press.

20. Morais S., Cabral A., Santos G., Madeira N. Melanoma brain metastates presenting as delirium: a case report. Arch Clin Psychiatry. 2017; 44(2): 53-54.

21. National Guidelines. Royal College of Psysicians. The prevention, diagnosis and management of delirium in older people. 2006.

22. Oldenbeuving A.W. (2013). Delirium in the acute phase after stroke. Doctoral Thesis, Utrecht University, Tilburg, the Netherlands.

23. Rabins P.V., Folstein M.F. Delirium and dementia: diagnostic criteria and fatality rates. Br J Psyciatry. 1982; 140: 149-153.

24. Robertsson B., Blennow K., Gottfries C.G. Delirium in dementia. Int J Geriatr Psychiatry. 1998; 13: 49-56.

25. Sanders R.D. Delirium, Neurotransmission, and Network Connectivity: The Search for a Comprehensive Pathogenic Framework. Anesthesiology. 2013; 118: 494-496.

26. Siddiqi N., House A.O., Holmes J.D. Occurrence and outcome of delirium in medical in-patients: a systematic literature review. Age Ageing. 2006; 35: 350-364.

27. Shang A.Z., Shen Q., Cordato D., Shen Q., Zhang Y., Chan D.K.Y. Delirium within three days of stroke in a cohort of elderly patients. JAGS. 2006; 54: 1192-1198.

28. Trzepacz P.T. Is there a common neural pathway in delirium? Focus on acetylcholine and dopamine. Semin Clin Neuropsyciatry. 2000; 5: $132-148$.

29. Trzepacz P.T., van der Mast R. The Neuropathophysiology of Delirium. Oxford: Oxford University Press; 2002.

30. van Sweden B., Mellerio F. Toxic ictal delirium. Biol Psychiatry. 1989; 25: 449-458.

31. Votey S.R., Peters A.L., Hoffman J.R. Disorders of water metabolism: hyponatremia and hypernatremia. Emerg Med Clin North Am 1989; 7: 749.

32. Washington S.D., VanMeter J.W. Anterior-posterior connectivity within the default Mode Network increases during maturation. Int J Med Biol Front. 2015; 21(2): 207-218.

33. Weddington W.W. The mortality of delirium: an underappreciated problem? Psychosomatics. 1982; 23: 1232-1235.

34. White S. The neuropthogenesis of delirium. Rev Clin Gerontol. 2002; 12: 62-67.

35. Maldonado J.R. Neuropathogenesis of delirium: review of current etiologic theories and common pathways. Am J Geriatr Psychiatry. 2013 Dec. 21 (12):1190-222. 